\title{
Enzymatic degradation study of PLA-based composite scaffolds
}

https://doi.org/10.1515/rams-2020-0005

Received Sep 03, 2019; accepted Dec 11, 2019

\begin{abstract}
Disadvantages in the use of polylactic acid (PLA) as a base material for Tissue Engineering applications include the low osteoconductivity of this biomaterial, its acidic degradation and the deficient cellular adhesion on its surface. In order to counteract these drawbacks, calcium carbonate $\left(\mathrm{CaCO}_{3}\right)$ and $\beta$-tricalcium phosphate $\left(\mathrm{Ca}_{3}(\mathrm{PO})_{2}, \beta\right.$-TCP) were proposed in this work as additives of PLA-based support structures. Composite scaffolds (PLA: $\mathrm{CaCO}_{3}: \beta$-TCP 95:2.5:2.5) manufactured by fused deposition modeling (FDM) were tested under enzymatic degradation using proteinase $K$ enzymes to assess the modification of their properties in comparison with neat PLA scaffolds. The samples were characterized before and after the degradation test by optical microscopy, scanning electron microscopy, compression testing and thermogravimetric and calorimetric analysis. According to the results, the combination of the PLA matrix with the proposed additives increases the degradation rate of the $3 \mathrm{D}$ printed scaffolds, which is an advantage for the application of the composite scaffold in the field of Tissue Engineering. The higher degradation rate of the composite scaffolds could be explained by the release of the additive particles and the statistically higher microporosity of these samples compared to the neat PLA ones.
\end{abstract}

Keywords: polylactic acid (PLA); bone tissue engineering; proteinase $\mathrm{K}$

\footnotetext{
*Corresponding Author: Ricardo Donate: Departamento de In-

*Corresponding Author: Ricardo Donate: Departamento de In-
geniería Mecánica, Universidad de Las Palmas de Gran Canaria, Campus Universitario de Tafira s/n, 35017, Las Palmas, Spain; Email: ricardo.donate@ulpgc.es; Tel.: +34-928-45-8603 Mario Monzón, Marıa Elena Alemán-Domınguez: Departamento de Ingeniería Mecánica, Universidad de Las Palmas de Gran Canaria, Campus Universitario de Tafira s/n, 35017, Las Palmas, Spain Zaida Ortega: Departamento de Ingeniería de Procesos, Universidad de Las Palmas de Gran Canaria, Campus Universitario de Tafira s/n, 35017, Las Palmas, Spain
}

¿ Open Access. () 2020 R. Donate et al., published by De Gruyter. (cc) BY 4.0 License

\section{Introduction}

Polylactic acid (PLA) is a widely used biomaterial in Bone Tissue Engineering because of its biocompatibility, its suitable mechanical properties and ease to be processed [1]. However, there are some disadvantages which limit its efficiency as a support material for bone ingrowth, such as the low osteoconductivity of this biomaterial, its acidic degradation and the deficient cellular adhesion on its surface [2]. Furthermore, the degradation of PLA is deemed to be too slow to enhance the replacement of the material by the new bone tissue [3, 4]. In order to counteract these drawbacks, calcium carbonate $\left(\mathrm{CaCO}_{3}\right)$ and $\beta$-tricalcium phosphate $\left(\mathrm{Ca}_{3}\left(\mathrm{PO}_{4}\right)_{2}, \beta\right.$-TCP) are proposed in this work as additives of PLA-based support structures intended for bone regeneration. $\beta$-TCP is a biodegradable and biocompatible ceramic material that has been extensively used in the field of Bone Tissue Engineering due to its osteoconductivity and its ability of complete bioresorption [5]. The use of the $\mathrm{CaCO}_{3}$ as an additive of PLA responds to the need of counteracting the release of acidic products during the degradation of the base material, maintaining the $\mathrm{pH}$ around 7.4 by buffer effect [6]. The composite blend developed was used to manufacture scaffolding structures by the method of additive manufacturing, under the category of "material extrusion” (ISO/ASTM 52900:2015), commonly known as fused deposition modeling (FDM). Additive manufacturing techniques provide the possibility of controlling the porosity of the scaffolds to be used in Tissue Engineering and personalizing their design according to the patients' needs [7].

In this work, the assessment of the degradation rate modification due to the presence of $\mathrm{CaCO}_{3}$ and $\beta$-TCP has been carried out. For this purpose, degradation tests of PLA and composite (PLA:CaCO ${ }_{3}: \beta$-TCP 95:2.5:2.5) scaffolds catalyzed by proteinase $\mathrm{K}$ enzymes were carried out. The enzymatic degradation test was designed with the aim of accelerating the degradation of PLA and composite scaffolds, since it can take more than 6 months to obtain significant weight losses when the experiment is carried out using PBS as degradation medium $[8,9]$. Several examples are found in the literature about the use of this en- 
zyme to degrade PLA fibres, films or scaffolds, obtaining significant differences in terms of weight loss or mechanical properties in days or even hours [10-12]. Sheng et al. [13], for example, obtained weight losses of between 30$50 \%$ in only 5 days by studying the degradation of PLA scaffolds using proteinase K enzymes. Although these working conditions do not rigorously simulate the conditions for the degradation of the material in vivo, this experiment design allows us to compare the composite scaffolds manufactured with the neat PLA ones. The degradation study was complemented with the morphological, thermogravimetric and mechanical characterization of the samples, before and after degradation, in order to assess the modification of the PLA properties due to the incorporation of the additives.

\section{Experimental}

PLA L105 in powder form was kindly supplied by Corbion Purac. Commercial grade calcium carbonate 0179-500G with a particle size of $30 \mu \mathrm{m}$ was purchased from VWR, while $\beta$-tricalcium phosphate ( $\beta$-TCP) with a mean particle size of $45 \mu \mathrm{m}$ was kindly provided by the 3B's Research Group of Universidade do Minho. These three materials were mixed to obtain the following mixture (wt:wt): PLA: $\mathrm{CaCO}_{3}: \beta$-TCP 95:2.5:2.5.

PLA and composite filaments were obtained using a lab prototype extruder with an $8 \mathrm{~mm}$ screw, a $1.6 \mathrm{~mm}$ diameter nozzle tip and an L/D cylinder ratio of 10 . The working parameters included a rotating speed of $7 \mathrm{rpm}$ and a temperature set at $245^{\circ} \mathrm{C}$ for the thermal resistance (the measured temperature in the proximity of the nozzle tip was equal to $180^{\circ} \mathrm{C}$ ). The filaments needed to print the 3D structures were obtained after a final cooling stage using compressed air. A BQ Hephestos 23D printer (Spain) was used to manufacture scaffolds with a rectangular $0 / 90^{\circ}$ pattern, a diameter of $9.8 \mathrm{~mm}$ and a height of $7 \mathrm{~mm}$, resulting in a theoretical porosity of $50 \%$. A nozzle diameter of $0.4 \mathrm{~mm}$ was used to print the scaffolds and the printing parameters included a temperature of $215^{\circ} \mathrm{C}$ and a deposition speed of $40 \mathrm{~mm} / \mathrm{s}$.

For the enzymatic degradation study, PLA and composite scaffolds were tested for time periods up to 5 and 10 days. Four replicas per group and time period were used. After measuring the weight of the scaffolds using an analytical balance $( \pm 0.1 \mathrm{mg}$, A\&D Scales Gemini Series, GR-200, Germany), the samples were placed individually in a 24 well-plate and immersed in $2 \mathrm{~mL}$ of $0.05 \mathrm{M} \mathrm{pH}$ 8.6 Tris- $\mathrm{HCl}$ buffer solution containing $0.2 \mathrm{mg} / \mathrm{mL}$ of proteinase $\mathrm{K}$ from
Tritirachium album (Merck, Darmstadt, Germany) and 0.2 $\mathrm{mg} / \mathrm{mL}$ of sodium azide (Merck, Darmstadt, Germany). The degradation study was carried out at $37^{\circ} \mathrm{C}$ and the bufferenzyme solution was replaced daily in order to maintain a high enzymatic activity. PLA and composite scaffolds incubated without enzymes in Tris-HCl solution were also evaluated as control samples. After the time periods studied, the scaffolds were weighed again to calculate the mass loss.

Before and after the degradation test, the surface morphology of the scaffolds was evaluated by scanning electron microscopy (SEM; Hitachi TM 3030 at an acceleration voltage of $15 \mathrm{kV}$ ). In addition, the pore size of these structures was assessed as the distance between filaments, using an Olympus BX51 optical microscope for that purpose. The pore size was calculated as the average of 40 measures per group of samples. Furthermore, the following equation was used in order to estimate the porosity of the 3D printed scaffolds $[14,15]$ :

$$
\% \text { porosity }=100 \cdot\left(1-\rho_{a p} / \rho_{\text {bulk }}\right),
$$

Where $\rho_{a p}$ is the apparent density of the structure and $\rho_{\text {bulk }}$ is the density of the bulk material. The first one was calculated using the mass and dimensions of the 3D printed scaffolds of each group of samples studied. The density of the bulk material was determined by measuring the dimensions and mass of samples $(n=8)$ extracted from the PLA and composite extruded filaments.

Samples extracted from the degraded scaffolds were subjected to thermogravimetric analysis in a TGA/DSC 1 Mettler Toledo device. PLA in powder form as well as nondegraded PLA and composite scaffolds were also analysed. The samples $(n=4)$ were placed in aluminium crucibles and heated up to $385^{\circ} \mathrm{C}$ at a rate of $10^{\circ} \mathrm{C} / \mathrm{min}$. The thermal cycle was performed while working with a nitrogen flow of $10 \mathrm{~mL} / \mathrm{min}$. During the TGA testing and using the same thermal cycle, calorimetric data (heat flow curve) of each type of sample was obtained, from which the melting temperature and the melting enthalpy were estimated. The melting enthalpy values obtained were used to calculate the crystallinity of each group of samples according to the following equation [16]:

$$
\% \mathrm{Xc}=100 \cdot\left[\Delta H_{f} /\left(\Delta H_{f}^{\circ} \cdot W_{P L A}\right)\right],
$$

Where $\mathrm{Xc}$ is the degree of crystallinity, $\Delta H_{f}$ is the enthalpy of fusion of the sample, $\Delta H_{f}^{\circ}$ corresponds to the heat of fusion of $100 \%$ crystalline PLA and $W_{P L A}$ is the net weight fraction of the PLA in the sample tested. The value used for $\Delta H_{f}^{\circ}$ was $93.7 \mathrm{~J} / \mathrm{g}[17]$.

Regarding the mechanical characterization, the scaffolds degraded were tested by compression on an LIYI 
(LI-1065, Dongguan Liyi Environmental Technology Co., Ltd.,China) testing machine in displacement control mode. Crosshead speed was set at $1 \mathrm{~mm} / \mathrm{min}$. The compressive modulus was calculated according to ASTM D695-15 using the initial steepest straight-line portion of the loadstrain curve. These results were compared with the ones obtained by testing non-degraded PLA and composite scaffolds. Four replicas were used per group of samples.

Statistical analysis was performed using MATLAB software (MATLAB and Statistics Toolbox Release 2017a, The MathWorks, Inc., Natick, USA). The data obtained during this study were analysed by the Wilcoxon two-sided rank sum test when comparing two groups and by the KruskalWallis test when more than two groups were compared. The significance level was set to ${ }^{\star} \mathrm{p}<0.05,{ }^{\star \star} \mathrm{p}<0.01$ and $\star \star \star \mathrm{p}<0.001$ for statistically significant, highly statistically significant and very highly statistically significant differences, respectively. All the figures show the mean values of each group and their standard deviations are represented with error bars.

\section{Results and Discussion}

After 5 days of enzymatic degradation, the mean weight loss of the PLA scaffolds group was $8.0 \%$, while this value was equal to $13.4 \%$ for the group of composite samples. At the end of the experiment (day 10), the PLA and composite samples were degraded up to a $17.6 \%$ and a $22.7 \%$, respectively. As showed in Figure 1, a statically significant difference $(\mathrm{p}<0.05)$ in terms of weight loss was obtained when comparing each type of samples at the two time periods studied. The PLA and composite samples used as control (immersed in Tris-HCl buffer solution with sodium azide but without enzymes) showed no weight loss during the degradation test. According to these results, the degradation rate of both groups between days 5 and 10 was very similar, being $9.6 \%$ and $9.3 \%$ for the PLA and composite samples, respectively. Therefore, the greater weight loss obtained for the composite scaffolds can be attributed to an increased degradation rate for these samples during the first steps of the experiment.

SEM images of each group of scaffolds showed mesostructures with well-defined square shaped pores in an interconnected network, as presented in Figure 2. Unlike PLA samples, which showed a translucent appearance, scaffolds manufactured using composite filaments exhibited a whitish colour due to the presence of $\mathrm{CaCO}_{3}$ and $\beta$-TCP particles. No relevant differences in relation to the surface morphology of the filaments printed by FDM

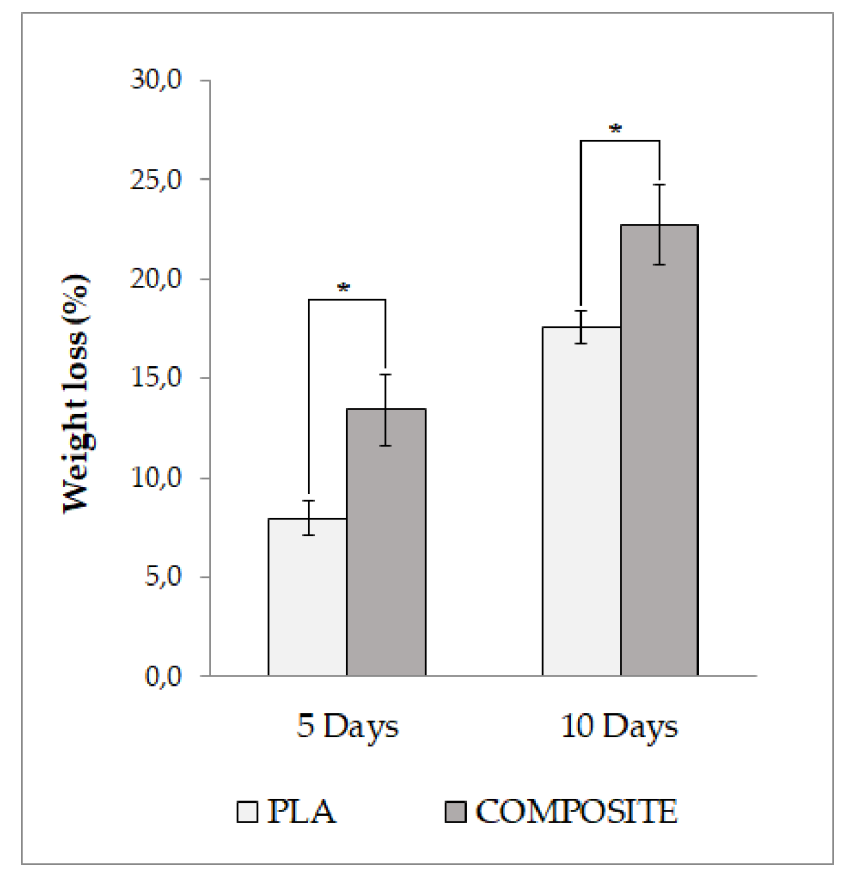

Figure 1: Weight loss percentage of 3D printed scaffolds at the time periods studied.

were observed when comparing PLA and composite samples before enzymatic degradation (Figures 2a and 2d). Both groups of samples showed filaments with a smooth surface but a slightly variable diameter. From the figures of the samples after 5 and 10 days of degradation (Figure 2b, $2 \mathrm{c}$ for PLA scaffolds and Figures 2e and $2 \mathrm{f}$ for the composite ones), it is evident how the diameter of the filaments is significantly reduced during the experiment, which led to an increase in the pore size and the porosity of the samples. These figures suggest different degradation profiles for the PLA and the composite samples, since for the latter group the presence of a large number of holes over the surface of the filaments can be easily observed; these voids could be attributed to the release of the additive particles during the degradation of the structure. This could be a reason for the higher rate of degradation observed in the composite scaffolds after 5 and 10 days. In the case of the PLA samples, some fractures on the surface of the filaments were found, from which the degradation of the structures progresses. We expect that the release of the additives during the degradation of the structure will have a positive effect on maintaining the $\mathrm{pH}$ at an appropriate level for the surrounding tissue.

Results concerning variations in porosity and pore size of the samples are presented in Table 1. Composite scaffolds showed a statistically higher porosity $(\mathrm{p}<0.05)$ in comparison with the neat PLA scaffolds before the degradation test. This result could be related to the greater varia- 
(a)

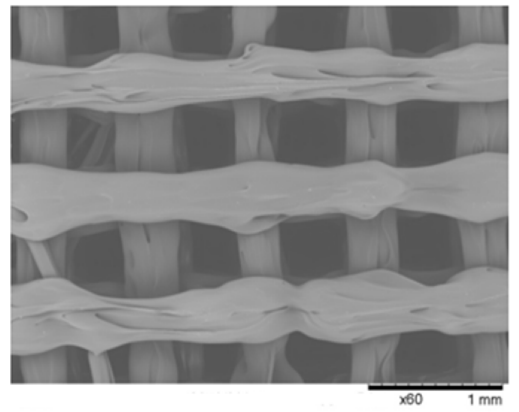

(d)

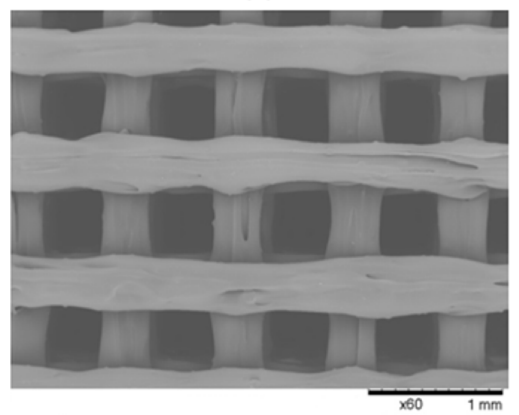

(b)

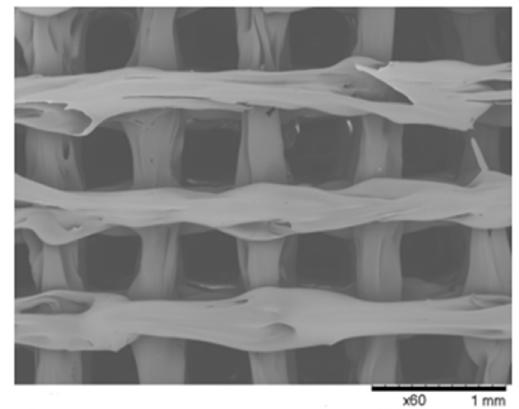

(e)

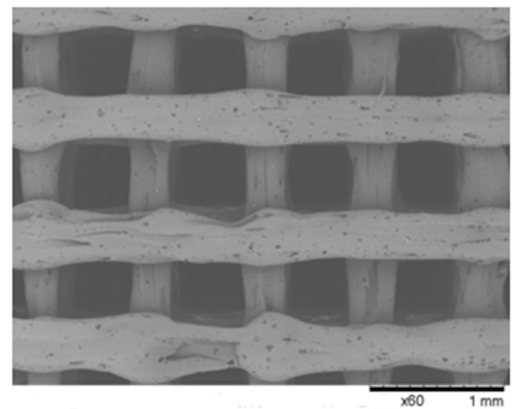

(c)

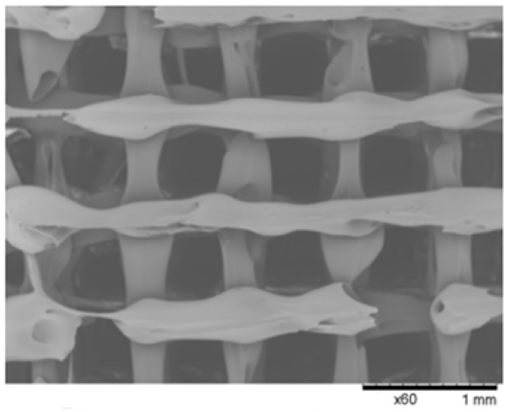

(f)

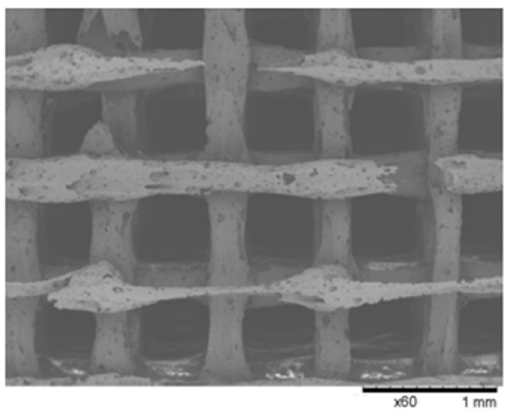

Figure 2: SEM images of the scaffolds analyzed: (a) PLA; (b) PLA 5D; (c) PLA 10D; (d) Composite; (e) Composite 5D; (f) Composite 10D. Scale bar: $1 \mathrm{~mm}$.

Table 1: Porosity and pore size values of the 3D printed scaffolds before and after degradation.

\begin{tabular}{ccc}
\hline Sample & Porosity $(\%)$ & Pore size $(\mu \mathrm{m})$ \\
\hline PLA & $57 \pm 2$ & $476 \pm 52$ \\
PLA 5D & $57 \pm 4$ & $531 \pm 51$ \\
PLA 10D & $65 \pm 3$ & $527 \pm 62$ \\
COMPOSITE & $62 \pm 2$ & $482 \pm 37$ \\
COMPOSITE 5D & $67 \pm 4$ & $476 \pm 58$ \\
COMPOSITE 10D & $68 \pm 4$ & $518 \pm 59$ \\
\hline
\end{tabular}

tion of the filaments diameter due to the presence of the additives. After 10 days of enzymatic degradation the mean porosity of the PLA samples was increased up to a $7.8 \%$, while the composite scaffolds final porosity was a $6.3 \%$ higher. The difference in porosity values before and after the test for the PLA samples was statistically significant $(\mathrm{p}<0.05)$. The increase of the porosity is accompanied with the increase of the pore size of the scaffolds, as the filament's diameter is decreased by the enzymes action. The higher pore size of the composite scaffolds could explain their enhanced degradation rate during the early stages of the experiment [13]. The differences in terms of pore size before and after 10 days of degradation test were statistically significant $(p<0.05)$ and very highly statistically sig- nificant $(\mathrm{p}<0.001)$ for PLA and composite samples, respectively.

The results obtained from the thermogravimetric and calorimetric analyses are shown in Table 2 , in which $\mathrm{T}_{\text {onset }}$ represents the temperature for the start of the thermal degradation of the material and $\mathrm{T}_{\text {peak }}$ is the temperature of maximum degradation rate. The comparison between the results of PLA powder and 3D printed PLA samples showed a statistically significant difference $(p<0.05)$ in terms of $\mathrm{T}_{\text {onset }}$, but not regarding the degree of crystallinity of the samples. One important trend observed (non-statistically significant difference) is the increase of the crystallinity due to the introduction of the additives into the PLA matrix, as the $\mathrm{CaCO}_{3}$ and $\beta$-TCP particles act as nucleation points [17].

On the other hand, after 10 days of enzymatic degradation PLA and composite samples showed, respectively, a statistically significant $(\mathrm{p}<0.05)$ and a highly statistically significant $(p<0.01)$ decrease in terms of degree of crystallinity when compared to the non-degraded PLA and composite samples. A statistically significant $(p<0.05)$ decrease was also obtained for the composite scaffolds after 10 days regarding the degradation and melting temperatures. The decrease of the samples' crystallinity was an unexpected result, as early enzymatic degradation preferentially occurs in the amorphous region of PLA [19], so 
Table 2: Values determined from TGA and calorimetric analysis.

\begin{tabular}{|c|c|c|c|c|c|}
\hline Sample & $T_{\text {onset }}\left({ }^{\circ} \mathrm{C}\right)$ & $T_{\text {peak }}\left({ }^{\prime} \mathrm{C}\right)$ & $\begin{array}{c}\text { Melting } \\
\text { temperature }(\cdot \mathrm{C})\end{array}$ & $\begin{array}{l}\text { Enthalpy of } \\
\text { fusion }(\mathrm{J} / \mathrm{g})\end{array}$ & Xc (\%) \\
\hline PLA powder & $350 \pm 1$ & $370 \pm 1$ & $174 \pm 2$ & $52.3 \pm 0.9$ & $55.9 \pm 1.0$ \\
\hline PLA & $353 \pm 1$ & $369 \pm 1$ & $175 \pm 3$ & $53.6 \pm 1.8$ & $57.2 \pm 1.9$ \\
\hline PLA 5D & $353 \pm 1$ & $369 \pm 1$ & $174 \pm 3$ & $38.5 \pm 2.9$ & $41.1 \pm 1.4$ \\
\hline PLA 10D & $350 \pm 2$ & $369 \pm 2$ & $174 \pm 3$ & $37.4 \pm 1.3$ & $40.0 \pm 1.4$ \\
\hline COMPOSITE & $353 \pm 1$ & $370 \pm 1$ & $177 \pm 3$ & $58.4 \pm 2.0$ & $59.3 \pm 2.0$ \\
\hline COMPOSITE 5D & $348 \pm 1$ & $368 \pm 1$ & $173 \pm 3$ & $35.4 \pm 1.2$ & $35.9 \pm 1.2$ \\
\hline COMPOSITE 10D & $349 \pm 1$ & $367 \pm 2$ & $171 \pm 2$ & $28.6 \pm 4.0$ & $29.0 \pm 1.0$ \\
\hline
\end{tabular}

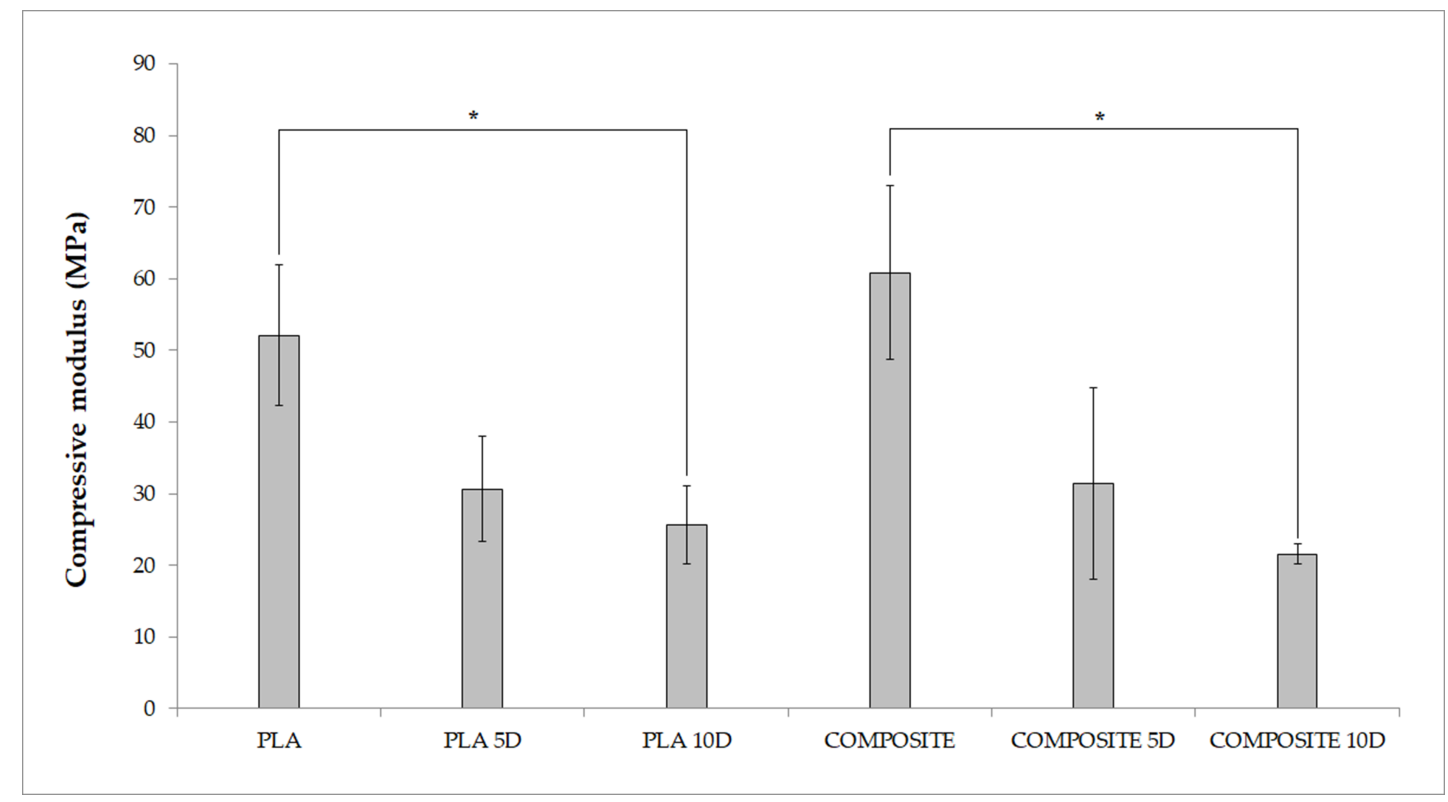

Figure 3: Mechanical properties of the 3D printed scaffolds under compression testing $\left({ }^{\star} p<0.05\right)$.

the crystallinity percentage should have increased during the experiment. However, according to the literature [4], when the molecular weight of the PLA samples under enzymatic degradation decreased down to a value around $30,000 \mathrm{~g} / \mathrm{mol}$, the hydrolytic degradation of crystalline regions of the material could be also enhanced. This can be a reason for the significant reduction of the crystallinity in both groups of samples, although further research is required to clarify this extent.

Regarding the mechanical characterization, the values of the compressive modulus for non-degraded samples were $52 \pm 10 \mathrm{MPa}$ for the PLA scaffolds and $61 \pm 12 \mathrm{MPa}$ for composite ones (Figure 3). After 5 days of degradation, these values were decreased down to $31 \pm 7 \mathrm{MPa}$ and $31 \pm 13$ $\mathrm{MPa}$, respectively. At the end of the experiment, the compression modulus was $26 \pm 5 \mathrm{MPa}$ for PLA scaffolds and $22 \pm 1$ MPa for the composite samples, both results being statisti- cally significantly lower $(\mathrm{p}<0.05)$ than the initial values for each type of sample.

\section{Conclusions}

The higher rate of degradation observed in the composite scaffolds after 5 and 10 days could be explained by the release of the additive particles and the statistically higher microporosity of composite scaffolds compared to the neat PLA samples, which could enhance the degradation rate at the early steps of the test. Regarding the compression test, a statistically significant decrease of the elastic modulus after 10 days of enzymatic degradation was confirmed for both PLA and composite scaffolds, with results still in the range of values reported for cancellous bone $(20-500 \mathrm{MPa})$ [20]. 
The combination of the PLA matrix with the proposed additives increases the degradation rate of the 3D printed scaffolds, which is an advantage for the application of the composite scaffold in the field of tissue engineering, taking into account the large amount of time necessary for the degradation of PLA structures. The ratio of use of these additives could be adjusted to the degradation rate required to match the growth rate of new bone tissue.

Acknowledgement: This contribution is part of the project BioAM (DPI2017-88465-R) funded by the Science, Innovation and Universities Spanish Ministry. Also, Ricardo Donate express his gratitude for the funding through the $\mathrm{PhD}$ grant program co-financed by the Canarian Agency for Research, Innovation and Information Society of the Canary Islands Regional Council for Employment, Industry, Commerce and Knowledge and by the European Social Fund (ESF) Canary Islands Integrated Operational Program 2014-2020, Axis 3 Priority Theme 74 (85\%). Grant code: TESIS2017010036.

\section{References}

[1] Narayanan, G., V. N. Vernekar, E. L. Kuyinu, and C. T. Laurencin. Poly (lactic acid)-based biomaterials for orthopaedic regenerative engineering. Advanced Drug Delivery Reviews, Vol. 107, 2016, pp. 247-276.

[2] Abert, J., A. Amella, S. Weigelt, and H. Fischer. Degradation and swelling issues of poly-(d,l-lactide) $/ \beta$-tricalcium phosphate/calcium carbonate composites for bone replacement. Journal of the Mechanical Behavior of Biomedical Materials, Vol. 54 , 2016, pp. 82-92.

[3] Saha, S. K., and H. Tsuji. Effects of molecular weight and small amounts of d-lactide units on hydrolytic degradation of poly(llactic acid)s. Polymer Degradation \& Stability, Vol. 91, No. 8 , 2006, pp. 1665-1673.

[4] Mainil-Varlet, P., R. Curtis, and S. Gogolewski. Effect of in vivo and in vitro degradation on molecular and mechanical properties of various low-molecular-weight polylactides. Journal of Biomedical Materials Research, Vol. 36, No. 3, 1997, pp. 360-380.

[5] Laurence, J., P. M. Baptista, A. Atala, and M. Van Beusekom. Translating Regenerative Medicine to the Clinic. Elsevier Inc., 2015. https://doi.org/10.1016/C2013-0-15405-0.

[6] Schiller, C., and M. Epple. Carbonated calcium phosphates are suitable $\mathrm{pH}$-stabilising fillers for biodegradable polyesters. Biomaterials, Vol. 24, No. 12, 2003, pp. 2037-2043.

[7] Zhang, Y. S., K. Yue, J. Aleman, K. M. Moghaddam, S. M. Bakht, J. Yang, W. Jia, V. Dell'Erba, P. Assawes, S. R. Shin, M. R. Dokmeci, R. Oklu, and A. Khademhosseini. 3D Bioprinting for Tissue and Organ Fabrication. Annals of Biomedical Engineering, Vol. 45, No. 1, 2017, pp. 148-163.
[8] de Tayrac, R., S. Chentouf, H. Garreau, C. Braud, I. Guiraud, P. Boudeville, and $M$. Vert. In vitro degradation and in vivo biocompatibility of poly(lactic acid) mesh for soft tissue reinforcement in vaginal surgery. Journal of Biomedical Materials Research. Part B, Applied Biomaterials, Vol. 85, No. 2, 2008, pp. 529-536.

[9] Yuan, X., A. F. T. Mak, and K. Yao. Comparative observation of accelerated degradation of poly(L-lactic acid) fibres in phosphate buffered saline and a dilute alkaline solution. Polymer Degradation \& Stability, Vol. 75, No. 1, 2002, pp. 45-53.

[10] Zeng, J., X. Chen, Q. Liang, X. Xu, and X. Jing. Enzymatic degradation of poly(L-lactide) and poly(epsilon-caprolactone) electrospun fibers. Macromolecular Bioscience, Vol. 4, No. 12, 2004, pp. 1118-1125.

[11] Tsuji, H., and H. Muramatsu. Blends of aliphatic polyesters: V. Non-enzymatic and enzymatic hydrolysis of blends from hydrophobic poly(L-lactide) and hydrophilic poly(vinyl alcohol). Polymer Degradation \& Stability, Vol. 71, No. 3, 2001, pp. 403413.

[12] Liu, L., S. Li, H. Garreau, and M. Vert. Selective enzymatic degradations of poly(L-lactide) and poly(epsilon-caprolactone) blend films. Biomacromolecules, Vol. 1, No. 3, 2000, pp. 350-359.

[13] Sheng, S. J., X. Hu, F. Wang, Q. Y. Ma, and M. F. Gu. Mechanical and thermal property characterization of poly-l-lactide (PLLA) scaffold developed using pressure-controllable green foaming technology. Materials Science and Engineering C, Vol. 49, 2015, pp. 612-622.

[14] Domingos, M., F. Intranuovo, A. Gloria, R. Gristina, L. Ambrosio, P. J. Bártolo, and P. Favia. Improved osteoblast cell affinity on plasma-modified 3-D extruded PCL scaffolds. Acta Biomaterialia, Vol. 9, No. 4, 2013, pp. 5997-6005.

[15] Yang, G. H., M. Kim, and G. Kim. Additive-manufactured polycaprolactone scaffold consisting of innovatively designed microsized spiral struts for hard tissue regeneration. Biofabrication, Vol. 9, No. 1, 2016, id. 015005.

[16] Esposito Corcione, C., F. Gervaso, F. Scalera, F. Montagna, A. Sannino, and A. Maffezzoli. The feasibility of printing polylactic acid-nanohydroxyapatite composites using a low-cost fused deposition modeling 3D printer. Journal of Applied Polymer Science, Vol. 134, No. 13, 2017. https://doi.org/10.1002/app.44656.

[17] Garlotta, D. A literature review of poly(lactic acid). Journal of Polymers and the Environment, Vol. 9, No. 2, 2001, pp. 63-84.

[18] Alemán-Domínguez, M. E., E. Giusto, Z. Ortega, M. Tamaddon, A. N. Benítez, and C. Liu. Three-dimensional printed polycaprolactone-microcrystalline cellulose scaffolds. Journal of Biomedical Materials Research. Part B, Applied Biomaterials, Vol. 107, No. 3, 2019, pp. 521-528.

[19] Reeve, M. S., S. P. McCarthy, M. J. Downey, and R. A. Gross. Polylactide Stereochemistry: Effect on Enzymic Degradability. Macromolecules, Vol. 27, No. 3, 1994, pp. 825-831.

[20] Leong, K. F., C. K. Chua, N. Sudarmadji, and W. Y. Yeong. Engineering functionally graded tissue engineering scaffolds. Journal of the Mechanical Behavior of Biomedical Materials, Vol. 1, No. 2, 2008, pp. 140-152. 